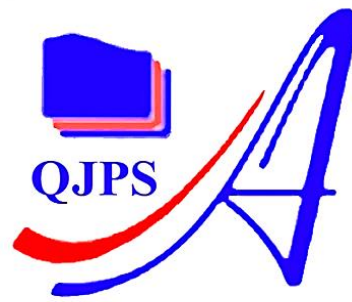

Al-Qadisiyah Journal of Pure Science

Al-Qadisiyah Journal of Pure Science

ISSN(Printed): 1997-2490 ISSN(Online): 2411-3514

DOI : /10.29350/jops.

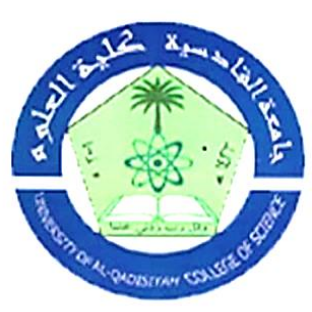

http://qu.edu.iq/iournalsc/index.php/IOPS

\title{
A Subclass of Harmonic Univalent Functions Defined by Salagean Integro Differential Operator
}

\section{Authors Names \\ a. Hasan BAYRAM}

Article History

Received on: 8/5/2020

Revised on: $4 / 6 / 2020$

Accepted on: 18/6/2020

Keywords:

Harmonic

Linear Operator

Univalent Functions

DOI:https://doi.org/10.29350/

jops.2020.25. 3. 1138

\section{ABSTRACT}

In this paper, we scrutinize some fundamental features of a subclass of harmonic functions defined by a new operator. Like coefficient inequalities, convex combinations, distortion bounds.

MSC: 30C45, 30C50

\section{Introduction}

Harmonic functions are a classic title in the class of geometric functions. Many researchers have studied these function classes from past to present, and since it has a wide range of applications, it is still a popular class. In this study, we will examinethe class of harmonic univalent functions, which is a subclass of harmonic functions. Let's show the open unit disk as $\mathbb{U}$. Let's show the family of continuous complex-valued harmonic functions that are harmonic in $\mathbb{U}$ as $\mathcal{H}$. $\mathcal{A}$ denotes the subclass of $\mathcal{H}$, including functions that are analytic in the open unit disk. $f$ be a harmonic function in $\mathbb{U}$ and $f$ may be written as $f=\mathfrak{h}+\overline{\mathfrak{g}}$; where $\mathfrak{h}$ and $\mathfrak{g}$ are in $\mathcal{A}$. We denominate $\mathfrak{h}$ is the analytic part of $f$, and $g$ is the co-analytic part of $f$. $\mathcal{S}$ denotes normalized analytic univalent functions in the open unit disk.

\section{a Department of Mathematics}


Clunie and Sheil-Small [5] proved that $f$ is sense-preserving and locally univalent in $\mathbb{U}$ if and only if $\left|\mathfrak{h}^{\prime}(z)\right|>$ $\left|\mathfrak{g}^{\prime}(z)\right|$. So no loss of generality we can write $\mathfrak{h}(0)=0$ and $\mathfrak{h}^{\prime}(0)=1$ since $\mathfrak{h}^{\prime}(z) \neq 0$.

In this way, writing

$$
\mathfrak{h}(z)=z+\sum_{k=2}^{\infty} a_{k} z^{k} \text { and } \mathfrak{g}(z)=\sum_{k=1}^{\infty} b_{k} z^{k}
$$

does not break generality.

Let $\mathcal{S H}$ indicate the family of functions $f=\mathfrak{h}+\overline{\mathfrak{g}}$ which are univalent, harmonic and sense-preserving in $\mathbb{U}$ for $f$ functions that provide $f(0)=f_{z}(0)-1=0$.

Consequently, $\mathcal{S H}$ includes the class of $\mathcal{S}$, which is normalized analytic univalent functions. Clearly, it will become apparent that although the analytic part $\mathfrak{h}$ of a function $f \in \mathcal{S H}$ is locally univalent, it need not be univalent. It can be easily demonstrated that the inequality of $\left|b_{1}\right|<1$ must be achieved in order to have sense-preserving property. The subclass $\delta \mathcal{H}^{0}$ of $\mathcal{S H}$ covers all functions in $\mathcal{S H}$ with $f_{\bar{z}}(0)=0$ property. Clunie and Sheil-Small [5] examined $\delta \mathcal{H}$ as well as its subclasses and discovered some coefficient bounds. Thenceforward, there have been various associated articles on $\mathcal{S H}$ and its subclasses. For more details see ([1], [3], [4], [6], [7], [8], [10], [11], [13], [14] and [15] etc.). Furthermore, notice that $\mathcal{S H}$ reduces to the class $\mathcal{S}$, if the co-analytic part of $f$ is identically zero.

For $f \in \mathcal{A}, n \in \mathbb{N}_{0}=\mathbb{N} \cup\{0\}$, the Salagean differential operator $D^{n}$ is defined by Salagean [9] $D^{n}: \mathcal{A} \rightarrow \mathcal{A}$,

$$
\begin{gathered}
D^{0} f(z)=f(z) \\
\ldots \\
D^{n+1} f(z)=z\left(D^{n} f(z)\right)^{\prime} .
\end{gathered}
$$

For

$$
f(z)=z+\sum_{k=2}^{\infty} a_{k} z^{k}
$$

we get

$$
D^{n} f(z)=z+\sum_{k=2}^{\infty} k^{n} a_{k} z^{k}
$$

This operator was developed and modified by many researchers over time. As a simple example for $f=\mathfrak{h}+\overline{\mathfrak{g}}$ as stated in (1), Jahangiri et al. [8] described the modified Salagean operator of $f$ like as

$$
\begin{gathered}
D^{0} f(z)=f(z), \\
\cdots \\
D^{n} f(z)=D^{n} \mathfrak{h}(z)+(-1)^{n} \overline{D^{n} \mathfrak{g}(z)},
\end{gathered}
$$

where

$$
D^{n} \mathfrak{h}(z)=z+\sum_{k=2}^{\infty} k^{n} a_{k} z^{k}
$$


and

$$
D^{n} \mathfrak{g}(z)=\sum_{k=1}^{\infty} k^{n} b_{k} z^{k}
$$

Salagean [9] defined a linear operator denoted by $I^{n}$ given by

$$
\begin{gathered}
I^{0} f(z)=f(z), \\
I^{\prime} f(z)=\frac{1}{z} \int_{0}^{z} \frac{f(t)}{t} d t \\
\ldots \\
I^{n} f(z)=I\left(I^{n-1} f(z)\right),
\end{gathered}
$$

where $f(z) \in \mathcal{A}, z \in \mathbb{U}, n \in \mathbb{N}_{0}$ and

$$
f(z)=z+\sum_{k=2}^{\infty} a_{k} z^{k}
$$

then

$$
I^{n} f(z)=z+\sum_{k=2}^{\infty} \frac{a_{k}}{k^{n}} z^{k} .
$$

Then Pall-Szabo [2] defined a linear operator for $z \in \mathbb{U}, \lambda \geq 0, n \in \mathbb{N}_{0}$ denoted by $\mathcal{L}^{n}$ given by $\mathcal{L}^{n}: \mathcal{A} \rightarrow \mathcal{A}$,

$$
\mathcal{L}^{n} f(z)=(1-\lambda) D^{n} f(z)+\lambda I^{n} f(z) .
$$

Therefore, if $f(z) \in \mathcal{A}$ and

$$
f(z)=z+\sum_{k=2}^{\infty} a_{k} z^{k}
$$

then

$$
\mathcal{L}^{n} f(z)=z+\sum_{k=2}^{\infty}\left[k^{n}(1-\lambda)+\lambda \frac{1}{k^{n}}\right] a_{k} z^{k} .
$$

Now we define the Salagean integro differential operator for functions $f=h+\bar{g}$ as stated in (1),

$$
\begin{aligned}
\mathcal{L}^{0} f(z) & =(1-\lambda) D^{0} f(z)+\lambda I^{0} f(z) \\
& \cdots \\
\mathcal{L}^{n} f(z)= & (1-\lambda) D^{n} f(z)+\lambda I^{n} f(z) \\
= & (1-\lambda)\left(D^{n} \mathfrak{h}(z)+(-1)^{n} \overline{D^{n} \mathfrak{g}(z)}\right)+\lambda\left(I^{n} \mathfrak{h}(z)+(-1)^{n} \overline{I^{n} \mathfrak{g}(z)}\right),
\end{aligned}
$$

where $n \in \mathbb{N}_{0}, 0 \leq \lambda \leq 1$. Therefore 


$$
\mathcal{L}^{n} f(z)=z+\sum_{k=2}^{\infty}\left[k^{n}(1-\lambda)+\lambda \frac{1}{k^{n}}\right] a_{k} z^{k}+(-1)^{n} \sum_{k=1}^{\infty}\left[k^{n}(1+\lambda)+\lambda \frac{1}{k^{n}}\right] \overline{b_{k} z^{k}} .
$$

Let show the subclass of $\mathcal{S H}$ containing functions $f$ as stated in (1) which provide the following condition as $\mathcal{S H}(\lambda, n, \mu)$

$$
\operatorname{Re}\left(\frac{\mathcal{L}^{n+1} f(z)}{\mathcal{L}^{n} f(z)}\right) \geq \mu, \quad 0 \leq \mu<1
$$

where $\mathcal{L}^{n} f(z)$, as stated in (2).

We denote the subclass $\overline{\mathcal{H H}}(\lambda, n, \mu)$ containing harmonic functions $f_{n}=\mathfrak{h}+\overline{\mathfrak{g}_{n}}$ in $\mathcal{S H}$ so that $h$ and $g_{n}$ be defined as follows

$$
\mathfrak{h}(z)=z-\sum_{k=2}^{\infty} a_{k} z^{k}, \quad \mathfrak{g}_{n}(z)=(-1)^{n} \sum_{k=1}^{\infty} b_{k} z^{k}
$$

$a_{k}, b_{k} \geq 0$.

If the parameters are chosen specially, $\mathcal{S H}(\lambda, n, \mu)$ classes are reduced to different subclasses of harmonic univalent functions (For example Jahangiri [7], Jahangiri et al. [8], Silverman [10], Silverman and Silvia [11], Uralegaddi and Somanatha [12], Cho and Srivastava [4], Bayram and Yalcin [13], Atshan and Wanas [16]).

\section{Main Results}

In Theorem 2.1, we present a sufficient coefficient condition for harmonic univalent functions that are the members of $\mathcal{S \mathcal { H } ^ { 0 }}(\lambda, n, \mu)$.

\subsection{Theorem}

Let $f=\mathfrak{h}+\overline{\mathfrak{g}}$. In here let $\mathfrak{h}$ and $\mathfrak{g}$ are as stated in (1) with $b_{1}=0$. Let

$$
\sum_{k=2}^{\infty}\left[k^{n}(1-\lambda)+\lambda \frac{1}{k^{n}}\right]\left(k^{n}(1-\lambda)+\lambda \frac{1}{k^{n}}-\mu\right)\left|a_{k}\right|+\sum_{k=2}^{\infty}\left[k^{n}(1+\lambda)+\lambda \frac{1}{k^{n}}\right]\left(k^{n}(1+\lambda)+\lambda \frac{1}{k^{n}}+\mu\right)\left|b_{k}\right| \leq 1-\mu,(6)
$$

where $n \in \mathbb{N}_{0}, 0 \leq \lambda \leq 1,0 \leq \mu<1$. Then $f$ is sense-preserving and harmonic univalent in $\mathbb{U}$ so we can say that $f \in \mathcal{S} \mathcal{H}^{0}(\lambda, n, \mu)$.

As a special notation for convenience, sometimes, we write

$$
A_{n}=\left[k^{n}(1-\lambda)+\lambda \frac{1}{k^{n}}\right]\left(k^{n}(1-\lambda)+\lambda \frac{1}{k^{n}}-\mu\right)
$$

and

$$
B_{n}=\left[k^{n}(1+\lambda)+\lambda \frac{1}{k^{n}}\right]\left(k^{n}(1+\lambda)+\lambda \frac{1}{k^{n}}+\mu\right)
$$

and

$$
C_{n}=k^{n}(1-\lambda)+\lambda \frac{1}{k^{n}} .
$$


in this article.

Proof. If we accept that $z_{1} \neq z_{2}$, we obtain

$$
\begin{aligned}
\left|\frac{f\left(z_{1}\right)-f\left(z_{2}\right)}{\mathfrak{h}\left(z_{1}\right)-\mathfrak{h}\left(z_{2}\right)}\right| & \geq 1-\left|\frac{\mathfrak{g}\left(z_{1}\right)-\mathfrak{g}\left(z_{2}\right)}{\mathfrak{h}\left(z_{1}\right)-\mathfrak{h}\left(z_{2}\right)}\right|=1-\left|\frac{\sum_{k=2}^{\infty} b_{k}\left(z_{1}^{k}-z_{1}^{k}\right)}{\left(z_{1}-z_{2}\right)+\sum_{k=2}^{\infty} a_{k}\left(z_{1}^{k}-z_{1}^{k}\right)}\right| \\
& >1-\frac{\sum_{k=2}^{\infty} k\left|b_{k}\right|}{1-\sum_{k=2}^{\infty} k\left|a_{k}\right|} \\
& \geq 1-\frac{\sum_{k=2}^{\infty} \frac{B_{n}}{1-\mu}\left|b_{k}\right|}{1-\sum_{k=2}^{\infty} \frac{A_{n}}{1-\mu}\left|a_{k}\right|} \geq 0
\end{aligned}
$$

that proving univalence. Pay attention $f$ is sense-preserving in $\mathbb{U}$. To show this feature;

$$
\begin{aligned}
\left|\mathfrak{h}^{\prime}(z)\right| & \geq 1-\sum_{k=2}^{\infty} k\left|a_{k}\right||z|^{k-1} \\
& >1-\sum_{k=2}^{\infty} \frac{A_{n}}{1-\mu}\left|a_{k}\right| \\
& \geq \sum_{k=2}^{\infty} \frac{B_{n}}{1-\mu}\left|b_{k}\right| \\
& >\sum_{k=2}^{\infty} k\left|b_{k}\right||z|^{k-1} \\
& \geq\left|\mathfrak{g}^{\prime}(z)\right| .
\end{aligned}
$$

Using the fact that Rew $\geq \alpha \Leftrightarrow|1-\alpha+w| \geq|1+\alpha-w|$, it suffices to show the following inequality

$$
\left|(1-\mu) \mathcal{L}^{n} f(z)+\mathcal{L}^{n+1} f(z)\right|-\left|(1+\mu) \mathcal{L}^{n} f(z)-\mathcal{L}^{n+1} f(z)\right| \geq 0 .
$$

Replacing for $\mathcal{L}^{n} f(z)$ and $\mathcal{L}^{n+1} f(z)$ in (7), we obtain

$$
\begin{gathered}
\left|(1-\mu) \mathcal{L}^{n} f(z)+\mathcal{L}^{n+1} f(z)\right|-\left|(1+\mu) \mathcal{L}^{n} f(z)-\mathcal{L}^{n+1} f(z)\right| \\
\geq 2(1-\mu)|z|-\sum_{k=2}^{\infty} C_{n}\left(C_{n}+1-\mu\right)\left|a_{k}\right||z|^{k}-\sum_{k=2}^{\infty} C_{n}\left(C_{n}-1+\mu\right)\left|b_{k}\right||z|^{k} \\
-\sum_{k=2}^{\infty} C_{n}\left(C_{n}-1-\mu\right)\left|a_{k}\right||z|^{k}-\sum_{k=2}^{\infty} C_{n}\left(C_{n}+1+\mu\right)\left|b_{k}\right||z|^{k} \\
>2(1-\mu)|z|\left\{1-\sum_{k=2}^{\infty} \frac{A_{n}\left|a_{k}\right|}{1-\mu}-\sum_{k=2}^{\infty} \frac{B_{n}\left|b_{k}\right|}{1-\mu}\right\} .
\end{gathered}
$$

This last phrase is not negative with (6), and therefore the proof is complete. 


\subsection{Theorem}

Let $f_{n}=\mathfrak{h}+\overline{\mathfrak{g}_{n}}$ be as stated in (4) with $b_{1}=0$. Then $f_{n} \in \overline{\mathcal{S H}}^{0}(\lambda, n, \mu)$, if and only if

$$
\sum_{k=2}^{\infty} A_{n}\left|a_{k}\right|+\sum_{k=2}^{\infty} B_{n}\left|b_{k}\right| \leq 1-\mu
$$

where $n \in \mathbb{N}_{0}, 0 \leq \lambda \leq 1,0 \leq \mu<1$.

Proof. Notice that $\overline{\delta \mathcal{H}}^{0}(\lambda, n, \mu)$ is a subclass of $\delta \mathcal{H}^{0}(\lambda, n, \mu)$. Then the "if" part can be easily proved from 2.1 Theorem. For the "only if" part, we must demonstrate that $f_{n} \notin \overline{\mathcal{S H}}^{0}(\lambda, n, \mu)$ if the inequality (8) is not valid. Here is an issue to be considered; a necessary and sufficient condition for $f_{n}=\mathfrak{h}+\overline{\mathfrak{g}_{n}}$ be as stated in (5), to be in $\overline{\mathcal{S H}}^{0}(\lambda, n, \mu)$ is that the condition (4) must be provided.

The above stipulation must be valid for all $z$ complex numbers where, $|z|=r<1$. After selecting the $z$ values from the positive real axis where $0 \leq|z|=r<1$, we obtain

$$
\operatorname{Re}\left\{\frac{(1-\mu) z-\sum_{k=2}^{\infty} A_{n} a_{k} z^{k}-\sum_{k=2}^{\infty} B_{n} b_{k} \bar{z}^{k}}{z-\sum_{k=2}^{\infty} C_{n} a_{k} z^{k}+\sum_{k=2}^{\infty} C_{n} b_{k} \bar{z}^{k}}\right\} \geq 0 .
$$

This is equivalent to

$$
\left\{\frac{(1-\mu)-\sum_{k=2}^{\infty} A_{n} a_{k} r^{k-1}-\sum_{k=2}^{\infty} B_{n} b_{k} r^{k-1}}{1-\sum_{k=2}^{\infty} C_{n} a_{k} r^{k-1}+\sum_{k=2}^{\infty} C_{n} b_{k} r^{k-1}}\right\} \geq 0 .
$$

If the inequality (8) is not valid, then the expression in (9) is negative for $r$ values approaching 1 . Hence there exists $z_{0}=r_{0}$ in $(0,1)$ for which the quotient in (9) is negative. This shows the required condition for $f_{n} \in \overline{\mathcal{S H}}^{0}(\lambda, n, \mu)$ and so the proof is complete.

\subsection{Theorem}

Let $f_{n}=\mathfrak{h}+\overline{\mathfrak{g}}_{n}$ be as stated in (5). At that case a necessary and sufficient condition for $f_{n} \in \overline{\mathcal{S H}}^{0}(\lambda, n, \mu)$ is

$$
f_{n}(z)=\sum_{k=1}^{\infty}\left(X_{k} h_{k}(z)+Y_{k} g_{n_{k}}(z)\right)
$$

where

$$
\mathfrak{h}_{1}(z)=z, \mathfrak{h}_{k}(z)=z-\frac{1-\mu}{A_{n}} z^{k}
$$

and

$$
\mathrm{g}_{n_{1}}(z)=z, \mathrm{~g}_{n_{k}}(z)=z+(-1)^{n} \frac{1-\mu}{B_{n}} \bar{z}^{k}
$$

for $X_{k} \geq 0, Y_{k} \geq 0, \sum_{k=1}^{\infty}\left(X_{k}+Y_{k}\right)=1, k=2,3, \cdots, n \in \mathbb{N}_{0}, 0 \leq \lambda \leq 1,0 \leq \mu<1$.

Notably, the extreme points of $\overline{\mathcal{S H}}^{0}(\lambda, n, \mu)$ are $\left\{\mathfrak{h}_{k}\right\}$ and $\left\{\mathfrak{g}_{n_{k}}\right\}$.

Proof. For $f_{n}$ functions which are as stated in (5), we obtain 


$$
\begin{gathered}
f_{n}(z)=\sum_{k=1}^{\infty}\left(X_{k} \mathfrak{h}_{k}(z)+Y_{k} \mathfrak{g}_{n_{k}}(z)\right) \\
=\sum_{k=1}^{\infty}\left(X_{k}+Y_{k}\right) z-\sum_{k=2}^{\infty} \frac{1-\mu}{A_{n}} X_{k} z^{k}+(-1)^{n} \sum_{k=2}^{\infty} \frac{1-\mu}{B_{n}} Y_{k} \bar{z}^{k} .
\end{gathered}
$$

Then

$$
\begin{gathered}
\sum_{k=2}^{\infty} \frac{A_{n}}{1-\mu}\left(\frac{1-\mu}{A_{n}} X_{k}\right)+\sum_{k=2}^{\infty} \frac{B_{n}}{1-\mu}\left(\frac{1-\mu}{B_{n}} Y_{k}\right) \\
=\sum_{k=2}^{\infty} X_{k}+\sum_{k=2}^{\infty} Y_{k}=1-X_{1}-Y_{1} \leq 1
\end{gathered}
$$

and so $f_{n} \in \overline{\mathcal{S H}}^{0}(\lambda, n, \mu)$. Moreover, if $f_{n} \in \overline{\mathcal{S H}}^{0}(\lambda, n, \mu)$, then

$$
a_{k} \leq \frac{1-\mu}{A_{n}}
$$

and

$$
b_{k} \leq \frac{1-\mu}{B_{n}}
$$

Set

$$
\begin{aligned}
& X_{k}=\frac{A_{n}}{1-\mu} a_{k}, \\
& Y_{k}=\frac{B_{n}}{1-\mu} b_{k},
\end{aligned}
$$

for $k=2,3, \cdots$ and

$$
X_{1}+Y_{1}=1-\left(\sum_{k=2}^{\infty} X_{k}+Y_{k}\right)
$$

where $X_{k} \geq 0, Y_{k} \geq 0$. Therefore, in accordance with, we have

$$
\begin{gathered}
f_{n}(z)=\left(X_{1}+Y_{1}\right) z+\sum_{k=2}^{\infty}\left(X_{k} \mathfrak{h}_{k}(z)+Y_{k} \mathfrak{g}_{n_{k}}(z)\right) \\
=\sum_{k=1}^{\infty}\left(X_{k} \mathfrak{h}_{k}(z)+Y_{k} \mathfrak{g}_{n_{k}}(z)\right) .
\end{gathered}
$$

\subsection{Theorem}

Let $f_{n} \in \overline{\mathcal{H H}}^{0}(\lambda, n, \mu)$. Then for $|z|=r<1$ and $n \in \mathbb{N}_{0}, 0 \leq \lambda \leq 1,0 \leq \mu<1$, we have 


$$
r-\frac{1-\mu}{A_{n}} r^{2} \leq\left|f_{n}(z)\right| \leq r+\frac{1-\mu}{A_{n}} r^{2}
$$

Proof. Here, we will only prove the right side since the proving of the left side, and the right side of the inequality is very similar. Let $f_{n} \in \overline{\mathcal{S H}}^{0}(\lambda, n, \mu)$. Taking the absolute value of $f_{n}$ we can easily see

$$
\left|f_{n}(z)\right| \leq r+\sum_{k=2}^{\infty}\left(a_{k}+b_{k}\right) r^{k} \leq r+\frac{(1-\mu) r^{2}}{A_{n}} \sum_{k=2}^{\infty} \frac{A_{n}}{1-\mu}\left|a_{k}\right|+\frac{(1-\mu) r^{2}}{A_{n}} \sum_{k=2}^{\infty} \frac{B_{n}}{1-\mu}\left|b_{k}\right| \leq r+\frac{1-\mu}{A_{n}} r^{2} .
$$

The left side can be shown in a similar way.

\subsection{Theorem}

The class $\overline{\mathcal{H}}^{0}(\lambda, n, \mu)$ is closed under convex combination.

Proof. Let $f_{n_{j}} \in \overline{\mathcal{S H}}^{0}(\lambda, n, \mu)$ for Let $\mathrm{j}=1,2, \ldots$, where $f_{n_{j}}$ is given by

$$
f_{n_{j}}(z)=z-\sum_{k=2}^{\infty} a_{k_{j}} z^{k}+(-1)^{n} \sum_{k=2}^{\infty} b_{k_{j}} \bar{z}^{k}
$$

Then by (7),

$$
\sum_{k=2}^{\infty} \frac{A_{n}}{1-\mu} a_{k_{j}}+\sum_{k=2}^{\infty} \frac{B_{n}}{1-\mu} b_{k_{j}} \leq 1
$$

For $\sum_{j=1}^{\infty} p_{j}=1,0 \leq p_{j} \leq 1$, the convex combination of $f_{n_{j}}$ can be written as

$$
\sum_{j=1}^{\infty} p_{j} f_{n_{j}}(z)=z-\sum_{k=2}^{\infty}\left(\sum_{j=1}^{\infty} p_{j} a_{k_{j}}\right) z^{k}+(-1)^{n} \sum_{k=2}^{\infty}\left(\sum_{j=1}^{\infty} p_{j} b_{k_{j}}\right) \bar{z}^{k}
$$

Then by (10),

$$
\begin{gathered}
\sum_{k=2}^{\infty} \frac{A_{n}}{1-\mu}\left(\sum_{j=1}^{\infty} p_{j} a_{k_{j}}\right)+\sum_{k=2}^{\infty} \frac{B_{n}}{1-\mu}\left(\sum_{i=1}^{\infty} p_{j} b_{k_{j}}\right) \\
=\sum_{j=1}^{\infty} p_{j} \sum_{k=2}^{\infty} \frac{A_{n}}{1-\mu} a_{k_{j}}+\sum_{j=1}^{\infty} p_{j} \sum_{k=2}^{\infty} \frac{B_{n}}{1-\mu} b_{k_{j}} \\
\leq \sum_{j=1}^{\infty} p_{j}=1 .
\end{gathered}
$$

This is the situation required by inequality (8). In this way $\sum_{j=1}^{\infty} p_{j} f_{n_{j}}(z) \in \overline{\mathcal{S H}}^{0}(\lambda, n, \mu)$.

\section{References}

[1] A. Ebedian, S. Azizi, S. Yalçın, “Univalent harmonic mappings and Hardy Spaces”, Turkish J. Math. 43(1), 284-292, 2019.

[2] A. O. Pall-Szabo, "On a Class of Univalent Functions Defined by Salagean Integro Differential Operator," Miskolc Mathematical Notes, 19(2), 1095-1106, 2018. 
[3] Y. Avc1 and E. Zlotkiewicz,. “On harmonic univalent mappings,” Ann. Univ. Mariae Curie-Sklodowska Sect. A, 44, 1-7, 1990.

[4] N. E. Cho and H. M. Srivastava, "Argument estimates of certain analytic functions defined by a class of multiplier transformations," Math. Comput. Modelling, 37, 39-49, 2003.

[5] J. Clunie and T. Sheil-Small, "Harmonic univalent functions," Ann. Acad. Sci. Fenn. Ser. A I Math., 9, 3-25, 1984.

[6] T. M. Flett, "The dual of an inequality of Hardy and Littlewood and some related inequalities," J. Math. Anal. Appl., 38, 746-765, 1972.

[7] J. M. Jahangiri, "Harmonic functions starlike in the unit disk," J. Math. Anal. Appl., 235, 470-477, 1999.

[8] J. M. Jahangiri, G. Murugusundaramoorthy and K. Vijaya, "Salagean-type harmonic univalent functions," South J. Pure Appl. Math., 2, 77-82, 2002.

[9] G. S. Salagean, "Subclasses of univalent functions," Lecture Notes in Math. Springer- Verlag Heidelberg, 1013, 362-372, 1983.

[10] H. Silverman, "Harmonic univalent functions with negative coefficients," J. Math. Anal. Appl., 220, 283-289, 1998.

[11] H. Silverman and E. M. Silvia, "Subclasses of harmonic univalent functions," N. Z. J. Math., 28, 275-284, 1999.

[12] B.A Uralegaddi and C. Somanatha, "Certain classes of univalent functions," Current topics in analytic function theory, World Sci. Publishing, Singapore, (Edited by H.M. Srivastava and S. Owa), 371-374, 1992.

[13] H. Bayram and S. Yalcin, "A subclass of harmonic univalent functions defined by a linear operator," Palestine Journal of Mathematics, 6(2):320326, 2017.

[14] S. Yalçın, “A new class of Salagean-Type harmonic univalent functions”, Appl. Math. Lett. 18(2); 191-198, 2005.

[15] H. Bayram, S. Yalçın, “A New Subclass of Harmonic Univalent Functions Defined By A Linear Operator”, Trans. J. Math. Mech. 10(2), 63-70, 2018.

[16] W. G. Atshan and A. K. Wanas, “On a New Class of Harmonic Univalent Functions”, Mathematicki Vesink, 65(4), 555-564, 2013. 